\title{
Two-photon Polymerization as Method for the Fabrication of Large Scale Biomedical Scaffold Applications
}

\author{
Thomas STICHEL ${ }^{* 1, * 2}$, Bert HECHT ${ }^{* 2}$, Ruth HOUBERTZ ${ }^{* 1}$ and G.erhard SEXTL ${ }^{* 1, * 3}$ \\ ${ }^{* 1}$ Fraunhofer ISC, Neunerplatz 2, 97082 Würzburg, Germany \\ ${ }^{* 2}$ Nano-Optics \& Biophotonics Group, Physikalisches Institut, Lehrstuhl für Experimentelle Physik V, Julius-Maximilians- \\ Universität, Am Hubland, 97074 Würzburg, Germany \\ ${ }^{* 3}$ Lehrstuhl für Chemische Technologie der Materialsynthese, Röntgenring 11, 97070 Würzburg
}

\begin{abstract}
Two-photon polymerization (2PP) using ultra-short laser pulses is a well-known methodology for the 3D free-form fabrication of optical devices with resolutions down to $100 \mathrm{~nm}$. However, the structure dimensions have been restricted to quite small sizes, mainly due to limitations of the focussing optics and due to very long fabrication times. Therefore, the largescale fabrication of biomedical scaffold structures with dimensions in the mm-range still remains challenging.

Using a diode-pumped Ytterbium laser system emitting $325 \mathrm{fs}$ laser pulses at $515 \mathrm{~nm}$ after second harmonic generation we are able to write arbitrary 3D structures in inorganic-organic hybrid polymers (ORMOCER ${ }^{\circledR}$ s). Our setup is able to produce structures with mm extension normal to the substrate at a structural resolution of a few microns. In particular, a 3D porous inner structure can be provided, which is required for three-dimensional cell growth to support cell adhesion and proliferation. Scaffold structures were produced with different parameters, and they were characterized in order to demonstrate their potential concerning resolution and scaffold quality. It is found that not only the experimental setup, but also the substrate material plays an important role for the scaffold fabrication process and structural quality.
\end{abstract}

DOI:10.2961/jlmn.2010.03.0005

Keywords: Two-photon polymerization, two-photon absorption, rapid prototyping, inorganic-

organic hybrid polymers, femtosecond pulse, scaffolds, micro-structuring

\section{Introduction}

Concerning high-resolution three-dimensional laser microfabrication, the two-photon polymerization (2PP) technique using femtosecond laser pulses with photosensitive materials is the state-of-the-art method up to date [1-4]. The physical principle is based on the two-photon absorption (TPA) phenomena which was theoretically postulated by Maria Göppert-Mayer in 1931 [5]. Her thesis describes the probability of the transition of an electron from the ground state to an excited state by the simultaneous absorption of two photons, a process which only occurs with significant probability at very high photon density. The 2PP method uses this effect by triggering a polymerization reaction with TPA which occurs when a fs laser beam is tightly focused into a photosensitive resin (which is transparent for the incident light). TPA shows quadratic dependence on the photon density and a corresponding threshold character. As a result, very small elliptical solid regions (so called voxels, i.e. volume pixels) are created in the liquid resin, in principle allowing the realization of very small structures. Previous publications report on minimum structure sizes around or below $100 \mathrm{~nm}$ [1-4], far beneath the diffraction limit of the employed wavelength. So far, no other competitive 3D microfabrication technique allows the fabrication of such highly resolved structures and consequently, 2PP is of potential interest for a large spectrum of applications which either profit from downsizing the dimensions of devices or need the precise control of complex 3D structures and substructures.

Important applications of 2PP are in the optical and optoelectronic sector. The fabrication of photonic crystals was demonstrated in many reports $[2,3,6]$ requiring high spatial resolution and precise control of structural parameters during the processing of the complicated periodical micro- structures. Another application sector is the field of biomedicine which recently has attracted considerable attention. Micro needle fabrication for drug delivery as well as the realization of scaffold structures with $2 \mathrm{PP}$ technology was demonstrated [7-9]. The idea behind scaffolds for medical applications is to obtain three-dimensional structures with distinct shapes that exhibit pores and holes, and therefore mimic the properties of extracellular matrices. Such artificial matrices are supposed to support threedimensional cell formation, cell proliferation, and differentiation in order to create neo-tissue or grafts from autologous cell cultures. The appropriate pore sizes should be in the range of a few micrometers to several hundreds of micrometers, dimensions which are easily achieved using $2 \mathrm{PP}$. But on the other hand, large samples have not yet been realized basically due to two drawbacks of the 2PP technique. First of all, the sample height in a standard setup is restricted to a few hundred of microns due to the limited working distance of common high-NA (numerical aperture) focusing optics. In addition, the slow fabrication rate of such systems limits the possibilities for application. For example, the production of a $1 \mathrm{~cm}^{3}$ solid sample with usual parameters, like a grating of $1 \mu \mathrm{m}$, and a writing speed of $0.1 \mathrm{~mm} / \mathrm{s}$, would take about 300 years, which is clearly not acceptable. To compete against rapid prototyping (RP) methods such as stereolithography, micro laser sintering, and $3 \mathrm{D}$ printing [10-12], significant improvements have to be implemented in order to speed up the fabrication process and to significantly increase the sample height. Once improvements over the current state of the art are implemented, perspectives are excellent, because (i) 2PP is a real 3D fabrication method (in contrast to RP), and (ii) because of the absence of time consuming molding, wiping (stereolithography), and sintering processes. 


\section{Materials and methods}

Here, we demonstrate the first step in scaling up the 2PP technology. Our setup provides a long travel airbearing axis system and focusing optics with large working distances, which allows fast writing speeds, variable grating parameters, and a maximum structure height of several millimetres. With this setup, scaffold structures with lateral dimensions in the range of 0.5 to $5 \mathrm{~mm}$ and heights of 0.3 to $1.2 \mathrm{~mm}$ were fabricated with a lateral $(\mathrm{x}, \mathrm{y})$ resolution of around $1 \mu \mathrm{m}$ and vertical $(\mathrm{z})$ resolution of a few microns.

As a major improvement, a diode-pumped Ytterbium laser system was used, which provides a pulse duration of $325 \mathrm{fs}$ at a $10 \mathrm{MHz}$ repetition rate, a maximum single pulse energy of $250 \mathrm{~nJ}$, and an average output power of about 2.5 $\mathrm{W}$ at a wavelength of $1030 \mathrm{~nm}$. A software-controlled Acoustic Optic Modulator (AOM) allows the reduction of the repetition rate as well as fast blanking of the laser beam. By second harmonic generation, the desired application wavelength of $515 \mathrm{~nm}$ is obtained. The power control during fabrication is managed with a half-wave plate mounted on a computer-controlled rotary stage and a polarizing beam-splitter. After beam expansion of a factor of three, the light is focused by a medium-NA objective which is adjustable in z-direction by an air-bearing axis. The exposure strategy is displayed in Figure 1. The focus of the objective is placed within the material reservoir which is confined by two cover glasses with a thickness of $150 \mu \mathrm{m}$ each and a spacer of variable thickness. The reservoir can be scanned in the plane (x,y) by two further air-bearing stages. The maximum travel distances of the axes are $10 \mathrm{~cm}$ with a maximum speed of $300 \mathrm{~mm} / \mathrm{s}$ and an accuracy of about 10 $\mathrm{nm}$. The structuring starts at the lower glass which acts as supporting substrate for polymerized material. The structure is then subsequently built up layer by layer by moving the sample holder in the plane (x, y) and the objective in the vertical direction (z). Furthermore, online observation can be performed by using a CCD camera and a dichroic mirror.

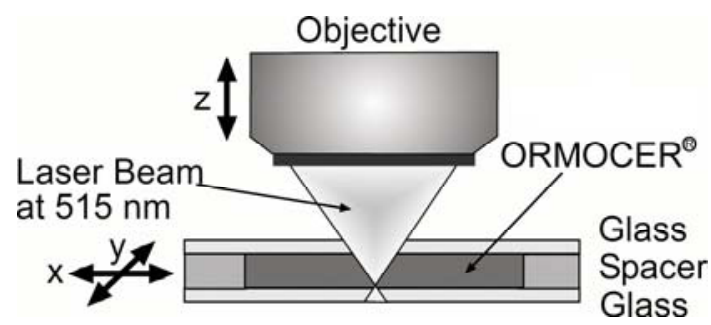

Fig. 1 2PP exposure strategy

The utilized resin material is ORMOCER ${ }^{\circledR}$, which is synthesized via hydrolysis/polycondensation reactions of alkoxysilane precursers. ORMOCER ${ }^{\circledR}$ s possess outstanding chemical, thermal, and mechanical stability, and they can be adapted to distinct applications in optics, micro- and polymer electronics as well as micromedicine by variation of the chemical constitution. A typical ORMOCER ${ }^{\circledR}$ consists of an inorganic Si-O-Si backbone, modified by polymerizalbe organic moities such as acrylate, methacrylate, styryl, or epoxy groups, and also non-polymerizable functionalities. The organic cross-linking can be triggered with conventional UV lithography, cw lasers, and by femtosec- ond laser pulses which initiate a two-photon polymerization process. Therefore, photoinitiators as additives play an important role. The initiator forms radicals upon n-photon excitation, thus further initiating a radical chain polymerization [13] by tackling the organic cross-linkable groups. Finally, the non-irradiated liquid resin can be removed with developer solutions, leaving the polymerized structures adhered on the glass substrates. During this process shrinkage of $5-15 \%$ can be observed [14], which is caused by strong capillary force developing during the evaporative drying. The material which was used is an inorganicorganic hybrid polymer containing methacrylate groups as polymerizable moieties $\left(\mathrm{ORMOCER}^{\circledR}\right)$ which was formulated with an UV photoinitiator (Inil from BASF, formerly Ciba Geigy), showing good performance concerning photosensitivity.

\section{Structures fabricated by long-distance objectives}

The two objectives used were the Nikon CFI Super Plan Fluor ELWD 40XC with a NA of 0.60 and the Nikon CFI Super Plan Fluor ELWD 20XC with a NA of 0.45 . They are characterized by long working distances, which are about $3.8 \mathrm{~mm}$ (NA 0.60 ) and $8.2 \mathrm{~mm}$ (NA 0.45 ), respectively. Furthermore, they feature a correction collar which enables one to adapt them to different cover glass (CG) thicknesses $(0-2 \mathrm{~mm})$ in order to diminish spherical aberration effects. Spherical aberration is a result of mismatched refractive indices (air/glass/material) and leads to blurring of the focal light distribution. In our case, the refractive indices of glass and material are closer than the ones of air/glass. Thus, for a first approach, we have included the spacer thickness into the corrective adjustments.

The scaffold design models were created by CAD software and imported into the software as binary stl-file, a widely used format for Rapid Prototyping and computeraided manufacturing. Important parameters are the slice and hatch distances (which are the grating space in vertical and horizontal direction, respectively), the writing-speed $v$ as well as the laser exposure power $P$. Generally, in comparison to common setups, higher values of grating and writing-speeds are aspired to achieve faster fabrication times.

\subsection{Objective with a numerical aperture of 0.60}

This objective was used in combination with a spacer thickness of $1 \mathrm{~mm}$. The fabricated scaffolds are displayed in Figure 2. Each scaffold was designed to have a base diameter $d$ of $500 \mu \mathrm{m}$, a height $h$ of about $300 \mu \mathrm{m}$, and a pore size $s$ of $100 \mu \mathrm{m}$. The slice and the hatch distances were 5 $\mu \mathrm{m}, v$ was $2.5 \mathrm{~mm} / \mathrm{s}$, and the positioning speed was 50 $\mathrm{mm} / \mathrm{s}$ at most, which results in a fabrication time of about 10 min for each individual scaffold. $P$ was varied between 3 and $7 \mathrm{~mW}$ and two different CG-correction (CG-cor.) adjustments were used.

When comparing the scanning electron microscope (SEM) images of the scaffolds in Figure 2, clear differences can be observed. The vertical pore size $s_{\text {vertical }}$ of the scaffold in Figure 3 (d) is about twice the pore size of the scaffold in Figure 3 (c) in contrast to the horizontal pore size $s_{\text {horizontal }}$ which is nearly the same. This fact indicates a strong increase of the aspect ratio of the voxels with increasing $P$ and, thus, a clear loss in axial resolution. 

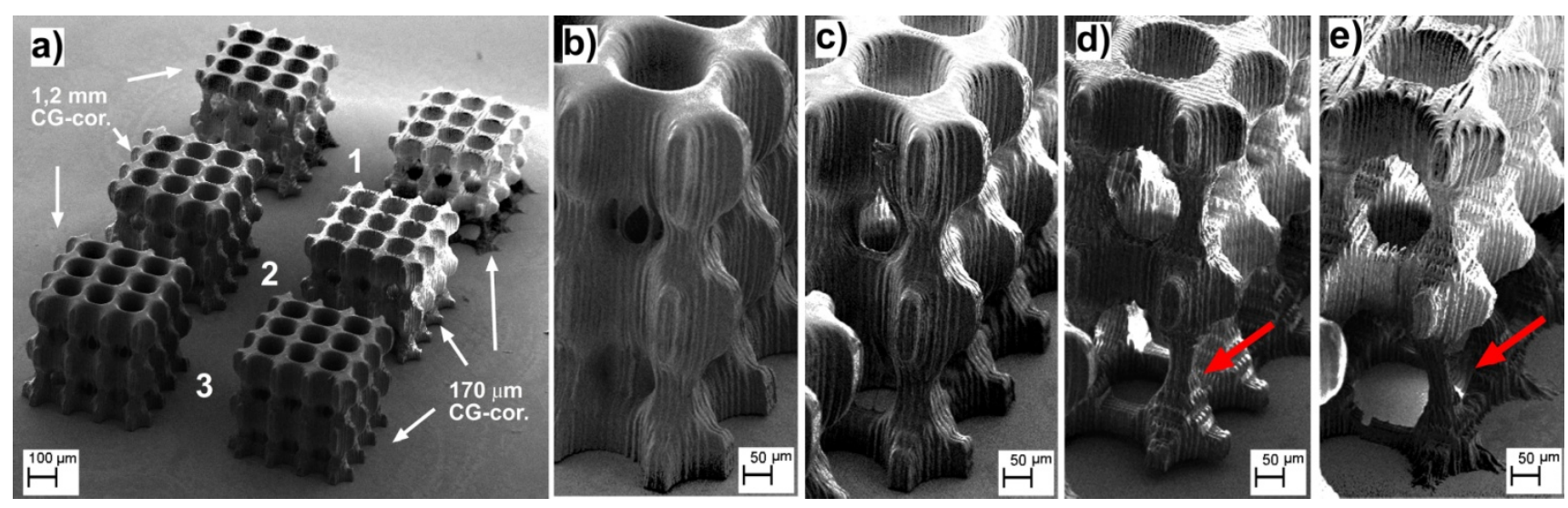

Fig. 2 SEM images of scaffolds $\left(500 \times 500 \times 300 \mu \mathrm{m}^{3}\right.$ ), produced in an ORMOCER ${ }^{\circledR}$ (formulated with 2 wt.- $\%$ Ini1) with a NA of 0.60. (a) $\mathrm{P}=3 \mathrm{~mW}$ (1), $5 \mathrm{~mW}$ (2), $7 \mathrm{~mW}$ (3). (b) $\mathrm{P}=7 \mathrm{~mW}$ (CG-cor. $1.2 \mathrm{~mm}$ ), (c) $\mathrm{P}=5 \mathrm{~mW}$ (CG-cor. $1.2 \mathrm{~mm}$ ), (d) $\mathrm{P}=3 \mathrm{~mW}$ (CG-cor. $1.2 \mathrm{~mm}$ ), and (e) $\mathrm{P}=3 \mathrm{~mW}$ (CG-cor. $150 \mu \mathrm{m})$.

Table 1

Dimensions of the scaffold in Figure 2d.

\begin{tabular}{cccc}
\hline & $\begin{array}{c}\text { 3D-model } \\
\text { data }\end{array}$ & $\begin{array}{c}\text { Scaffold } \\
\text { measurement }\end{array}$ & $\begin{array}{c}\text { Discrepancy } \\
\text { factor }\end{array}$ \\
\hline $\mathrm{d}$ & $500 \mu \mathrm{m}$ & ca. $420 \mu \mathrm{m}$ & 0.84 \\
$\mathrm{~h}$ & $300 \mu \mathrm{m}$ & ca. $445 \mu \mathrm{m}$ & 1.48 \\
$\mathrm{~S}_{\text {horizontal }}$ & $100 \mu \mathrm{m}$ & ca. $83 \mu \mathrm{m}$ & 0.83 \\
$\mathrm{~s}_{\text {vertical }}$ & $100 \mu \mathrm{m}$ & ca. $108 \mu \mathrm{m}$ & 1.08 \\
\hline
\end{tabular}

Table 2 Dimensions of the scaffold in Figure 3d.

\begin{tabular}{cccc}
\hline & $\begin{array}{c}\text { 3D-model } \\
\text { data }\end{array}$ & $\begin{array}{c}\text { Scaffold } \\
\text { measurement }\end{array}$ & $\begin{array}{c}\text { Discrepancy } \\
\text { factor }\end{array}$ \\
\hline $\mathrm{d}$ & $1000 \mu \mathrm{m}$ & ca. $850 \mu \mathrm{m}$ & 0.85 \\
$\mathrm{~h}$ & $1000 \mu \mathrm{m}$ & ca. $920 \mu \mathrm{m}$ & 0.92 \\
$\mathrm{~S}_{\text {horizontal }}$ & $230 \mu \mathrm{m}$ & ca. $190 \mu \mathrm{m}$ & 0.83 \\
$\mathrm{~s}_{\text {vertical }}$ & $230 \mu \mathrm{m}$ & ca. $175 \mu \mathrm{m}$ & 0.76 \\
\hline
\end{tabular}

For the scaffolds displayed in Figure 2 (d) and Figure 2 (e), differences between the lower pillars (arrows) of the structures processed with a CG-cor. of $1.2 \mathrm{~mm}$ and $150 \mu \mathrm{m}$ at the same P. For a CG-cor. of $1.2 \mathrm{~mm}$ the pillar is much more stable than for a CG-cor. of $170 \mu \mathrm{m}$, what points to minor blurring and a higher maximum photon intensity in the focus for a CG-cor. of $1.2 \mathrm{~mm}$ than for a CG-cor. of 170 $\mu \mathrm{m}$. The scaffold presented in Figure 2 (d) was examined in more detail by SEM. Table 1 presents the results in comparison to the $3 \mathrm{D}$ model data. It can be noticed that the vertical direction of the model is clearly stretched. The pores are elongated, and the total structure height exceeds $300 \mu \mathrm{m}$ by far. The reason for that is the refractive index step at the air/material interface leading to a strong defocusing effect. This means that the axial movement of the objective results in a larger shift of its focus and, consequently, the model will be stretched by a factor depending strongly on the objective's NA. For a NA of 0.60 , a factor of 1.72 for the elongation can be calculated, which would lead to a height of the structure of about $516 \mu \mathrm{m}$ and a vertical pore size of about $172 \mu \mathrm{m}$. If material shrinkage of
$16 \%$ is taken into account which is obtained by the discrepancy factor (ratio of scaffold measurement and 3D model data) of $d$ and shorizontal, a rough agreement with the calculation is found.

\subsection{Objective with a numerical aperture of 0.45}

An objective with a numerical aperture of 0.45 was used with a $2 \mathrm{~mm}$ thick spacer. Two different scaffold sizes were written. Each design contains cubic pore sizes of 230 x $230 \times 230 \mu \mathrm{m}^{3}(1 \times \mathrm{b} \mathrm{x} \mathrm{h})$, whereas the total structure sizes are different namely 1 x $1 \times 1 \mathrm{~mm}^{3}$ for the "small" scaffold and $5 \times 5 \times 1.2 \mathrm{~mm}^{3}$ for the "large" one.

The slice-distances were adjusted to be $15 \mu \mathrm{m}$, and the hatch distances were set to $20 \mu \mathrm{m}$. In order to avoid the defocusing effect, a software-based correction module was implemented into the application software, which calculates the stretch factor for given refractive indices and NAs, and corrects the axis movement automatically. For performance testing, a writing speed of $5 \mathrm{~mm} / \mathrm{s}$ ("small") and $2.5 \mathrm{~mm} / \mathrm{s}$ ("large") was chosen leading to fabrication times of about 5 min ("small") and 4 h 20 min ("large"), respectively.

The fabricated scaffolds in ORMOCER ${ }^{\circledR}$ are displayed in Figures 3 and 4. The "small" scaffolds were fabricated with different $P$. For an average laser power of $6 \mathrm{~mW}$, the scaffolds are instable. Especially the one which was fabricated without CG-cor. is strongly distorted indicating a low and insufficient degree of organic cross-linking. The dimensions of the scaffold of Figure 3 (d) were estimated analogously to the scaffold in Figure 2 (d) using SEM imaging. The results are listed in Table 2 . With the values $d$ and $s_{\text {hori- }}$ zontal, a material shrinkage of about $16 \%$ can be estimated. The discrepancy in $h$ and $s_{\text {vertical }}$ is based on the high aspect ratio of the voxels once again.

In Figure 4 (b), a zoom into the SEM image of the topview of the "large" scaffold is shown. The small pores of about $10 \mu \mathrm{m}$ in diameter were chosen in order to provide structures where cells might adhere, and their tube-like shapes are the result of the roughly chosen hatching distances. The large pores are big enough to allow an infiltration with cells The entire structure is estimated to have a very high porosity of about $80 \%$. 

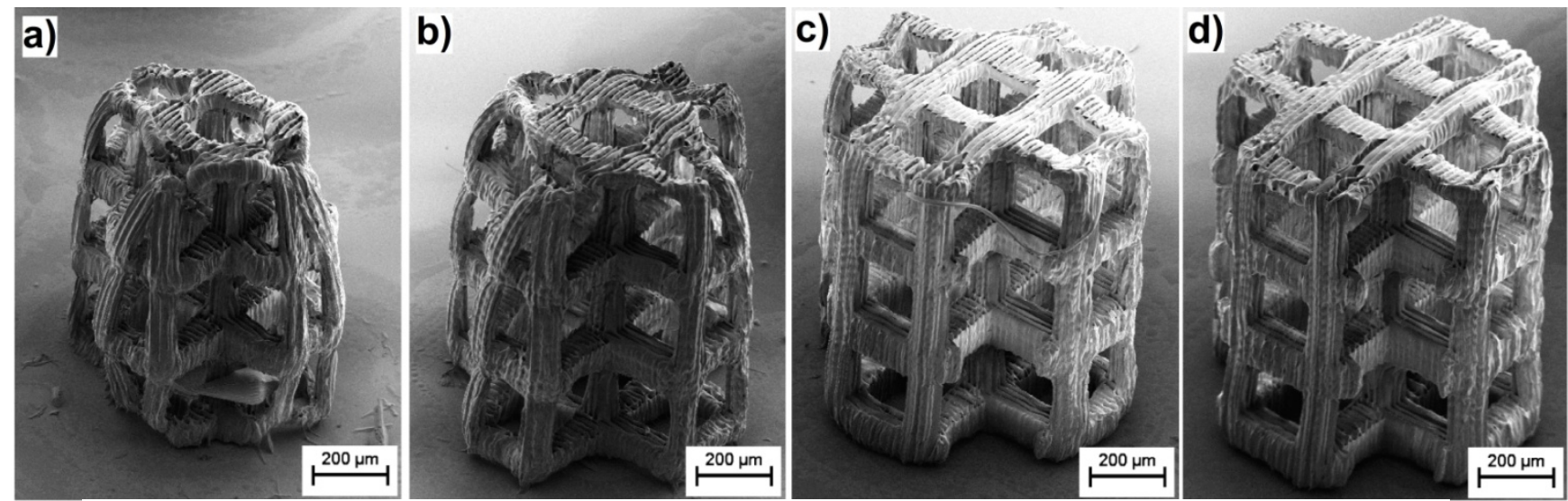

Fig. 3 SEM images of scaffolds $\left(1 \times 1 \times 1 \mathrm{~mm}^{3}\right)$, produced in an ORMOCER ${ }^{\circledR}$ (formulated with 2 wt.-\% Ini1) and a NA of 0.45. (a) $\mathrm{P}=6 \mathrm{~mW}$ (0 mm CG-cor.), (b) $\mathrm{P}=6 \mathrm{~mW}$ ( $2 \mathrm{~mm} \mathrm{CG-cor.),} \mathrm{(c)} \mathrm{P}=7 \mathrm{~mW}$ (2 mm CG-cor.), and (d) $\mathrm{P}=8 \mathrm{~mW}(2 \mathrm{~mm}$ CG-cor. $)$
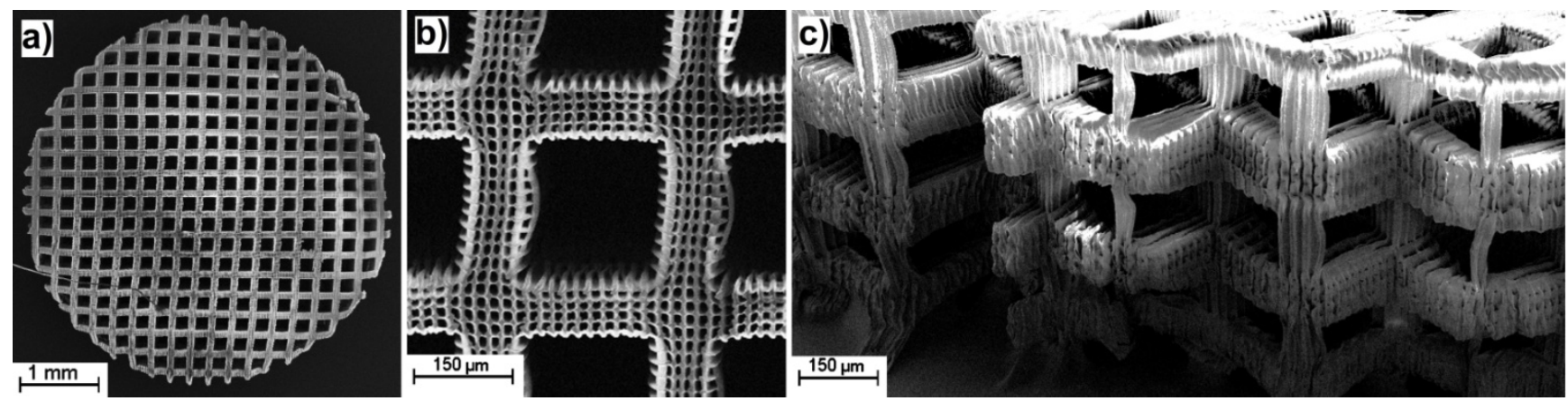

Fig. 4 SEM images of a scaffold $\left(5 \times 5 \times 1.2 \mathrm{~mm}^{3}\right)$, fabricated in ORMOCER ${ }^{\circledR}$ (formulated with 1 wt.-\% Ini1) with $\mathrm{P}=10 \mathrm{~mW}$, a NA of 0.45 , and a CG-cor. of $2 \mathrm{~mm}$. (a) Top view, (b) zoom into the structure, and (c) sideview.

\section{Conclusion and perspective}

We have demonstrated the first step of up-scaling the $2 \mathrm{PP}$ technology to create large-scale scaffolds for tissue engineering. The medium-NA optic allows the fabrication of highly porous structures with a height of several millimeters in an adequate fabrication time. Parameters were investigated which influence the results regarding mechanical stability, resolution perspectives and fabrication time. Obviously, the structure stability and degree of organic crosslinking are correlated. This will be further investigated by spectroscopic methods. First high resolution $\mu$-Raman measurements suggest a degree of organic cross-linking of 30 to $40 \%$ which is much lower compared to the results of common UV lithography and 2PP setups with the same material.

\section{Acknowledgments}

The authors would like to thank the German Science Foundation with the "sub-100-nm Program" (grant: HO 2475/31) and the Fraunhofer Gesellschaft für Angewandte Forschung e.V. (Challenge) for the financial support. Furthermore, the excellent technical support by our colleagues in Würzburg is greatfully acknowledged.

\section{References}

[1] S. Kawata, H.-B. Sun, T. Tanaka, K. Takada: Nature 412, (2001) 697-698
[2] J. Serbin, A. Egbert, A. Ostendorf, B.N. Chichkov, R. Houbertz, G. Domann, J. Schulz, C. Cronauer, L. Fröhlich, M. Popall: Opt. Lett, 28(5), (2003) 301-303.

[3] R. Houbertz: Appl. Surf. Sci. 247, (2005) 505.

[4] J. Li, B. Jia, and M. Gu: Opt. Express, 16(24), (2008) 20073-20080.

[5] M. Göppert-Mayer: Ann Phys. 9, (1931) 273-294.

[6] R. Houbertz, V. Satzinger, V. Schmid, W. Leeb, G. Langer: Proc of SPIE 7053, (2008) 70530B.

[7] A. Ostendorf, B. N. Chichkov: Photonics Spectra, October issue, (2006).

[8] R. J. Narayan, C. Jin, A. Doraiswamy, I. N. Mihailescu, M. Jelinek, A. Ovsianikov, B. Chichkov, D. B. Chrisey: Adv. Eng. Materials, 7(12), (2005) 1083-1098.

[9] A. Doriaswamy, T. Patz, R. Narayan, B. Chichkov, A. Ovsianikov, R. Houbertz, R. Modi, R. Auyeung, D.B. Chrisey: Mater. Res. Soc. Symp. Proc, 845, (2005) AA2.4.1.

[10] R. Landers, U. Hübner, R. Schmelzeisen, R. Mülhaupt: Biomaterials, 23, (2002) 4437-4447.

[11] T. Weigel, G. Schinkel, A. Lendlein: Expert Rev. Med. Devices, 3(6), (2006) 835-851.

[12] M. Lee, J. C. Y. Dunn, B. M. Wu: Biomaterials, 26, (2005) 4281-4289.

[13] G. Odian: "Principles of Polyermization", ed. by J. Wiley \& Sons, (New York, 1981)

[14] Y. Li, F. Qi, H. Yang, Q. Gong, X. Dong, X. Duan: Nanotechnology, 19, (2008) 055303. 